\title{
HIGH SIGNAL-TO-NOISE RATIO - THE SPECTROSCOPIC KEY TO ALGOL SYSTEMS
}

\author{
JOCELYN TOMKIN \\ Astronomy Department \\ University of Texas \\ Austin, Texas, 78712 \\ U.S.A.
}

(Received 20 October, 1988)

\begin{abstract}
The usefulness of high signal-to-noise-ratio spectra for both radial-velocity and abundance studies of Algol systems is emphasised. It is shown that division by a hot star is a worthwhile step in pursuit of this objective. A preliminary analysis of high signalto-noise-ratio, red and near-infrared, Reticon observations of $\mathrm{R}$ CMa shows that its primary has solar CNO abundances within the 0.3 dex observational error. The low-mass $\left(0.17 \mathrm{~m}_{\odot}\right)$ secondary of this Algol system must have lost a large fraction of its original mass. Some of this material would have been extensively processed during the secondary's main-sequence lifetime and would therefore have had a highly non-solar CNO-abundance distribution. The lack of serious contamination of the primary's abundances is consistent with most, but not all, plausible mass-transfer scenarios.
\end{abstract}

\section{Introduction}

When I first got into the binary business I was pretty naive. One of my misconceptions was that the main advantage of modern photodiode detectors for the detection of faint secondaries, which was my initial interest, is their red and near-infrared sensitivity. In the typical Algol system the secondary is cooler than the primary so the secondary spectrum is easier to detect in the red and near-infrared where it makes a larger contribution to the total light than in the blue.

Red and near-infrared sensitivity is important, however, it is not the key consideration. High signal-to-noise-ratio capability is what really distinguishes modern detectors from their photographic-plate predecessors.

In the context of detection and measurement of a faint secondary spectrum that contributes $5 \%$ or less, say, of the total light, this capability hardly needs to be stressed. But it may also be important for measurements of the primary spectrum, even though the primary may contribute nearly all of the light. Weak lines, for example, are very desirable for the purposes of abundance studies; they provide abundances that are free of the desaturation uncertainties that plague strong lines and are less affected by non-LTE than strong lines. The broad lines typical of Algol systems mean that these weak lines will also be very shallow. If we, somewhat arbitrarily, choose $70 \mathrm{~km} \mathrm{~s}^{-1}$ as a typical $\mathrm{v}$ sin i for an Algol-system primary and call a $60 \mathrm{~m} \AA$ line at a wavelength of $6000 \AA(\log W / \lambda=-5.0)$ a weak line, then the line will be $3 \AA$ wide and only $2 \%$ deep. (This is in the primary spectrum; the secondary spectrum's presence will make it even shallower.) Measurement of such a line's equivalent width clearly calls for high signal-to-noise ratio. 
In the remainder of this talk I will argue the merits of division by a hot star for realisation of modern detectors' full signal-to-noise ratio potential and will then present preliminary results of a CNO abundance analysis of R CMa.

\section{Hot-Star Division}

Figure 1 shows the spectrum of V505 $\mathrm{Sgr}(\mathrm{A} 2 \mathrm{~V}, \mathrm{~V}(\max )=6.48, \mathrm{P}=1 \mathrm{~d} 2)$, an Algol system whose secondary spectrum has hitherto not been detected. The observation was made with the McDonald Observatory $2.7-\mathrm{m}$ telescope, coude spectrograph and a Reticon detector (Vogt, Tull and Kelton 1978). The numerous telluric water lines, whose apparent strength is exaggerated by the expanded intensity scale, obscure the stellar features; neither the primary nor the secondary NaD lines are apparent.

Figure 2 shows the same observation after division by a hot star instead of a flat-field lamp. The hot-star division removes the telluric lines so that not only V505 Sgr's primary $\mathrm{NaD}$ lines but also its secondary $\mathrm{NaD}$ lines along with the sharp interstellar $\mathrm{NaD}$ lines are apparent. Although this observation was made in the summer (early September) when telluric water lines are seasonally strong, division by a hot star is worthwhile even in winter.

Figure 2 also shows that hot-star division is not always an unqualified blessing; features in the hot star's own spectrum are a source of potential complication. The hot star ( $\lambda$ Aql, $\mathrm{B} 8.5 \mathrm{~V}$ ) has a weak $5875 \AA \mathrm{HeI}$ line which is responsible for the bump at this wavelength. It may also have its own $\mathrm{NaD}$ lines, but they appear to be negligibly weak. There is no evidence of distortion of V505 Sgr's primary and secondary $\mathrm{NaD}$ lines by putative hot-star $\mathrm{NaD}$ lines.

Clearly telluric lines are a pest in the neighbourhood of the $\mathrm{NaD}$ lines, however, this region and a few others $\left(\sim 6300, \sim 6900\right.$, and $\sim 7600 \AA, \mathrm{O}_{2} ; 7900-8500 \AA, \mathrm{H}_{2} \mathrm{O} ; 9000-$ $9800 \AA, \mathrm{H}_{2} \mathrm{O}$ ) are the only parts of the red and near-infrared where one has to worry about them. For a long time this was my thinking. But the weaker the stellar features in question, the lower the threshold above which telluric lines become a potential nuisance. In fact telluric lines are so prevalent that if one plans to measure stellar lines of order $2 \%$ deep, or less, then the situation is reversed; starting at a wavelength of about $5700 \AA$ and going through the red and near-infrared, telluric-line-free wavelength intervals are the exception, not the rule.

Figure 3 shows part of a McDonald Reticon observation of the detached eclipsing binary $\alpha \mathrm{CrB}(\mathrm{AOV}+\mathrm{G}, \mathrm{V}(\max )=2.24, \mathrm{P}=17 \mathrm{~d} 3$ ). Although the observation is at a wavelength $(8800 \AA)$ not noted for telluric lines and although it was made in the winter when atmospheric water content is seasonally low, it is apparent that division by a hot star, instead of a flat-field lamp, cleans up the observation considerably. The very weak lines of the faint secondary, which are the features of interest, can be identified with more confidence and when the time comes to measure them one does not have to worry about dodging telluric lines. See Tomkin and Popper (1986) for an analysis of $\alpha \mathrm{CrB}$ which includes secondary radial velocities based on these lines.

The presence of specks of dirt or dust, which may have lodged on the detector's chip, provide another argument for hot-star division. The hot star illuminates the individual diodes in much the same way that the star does, whereas the flat-field lamp does not, so hot-star division removes dirt-on-chip irregularities much more effectively. The fringing that commonly affects CCD detectors, combined with similar star-illumination versus lamp-illumination considerations, is a further reason for hot-star division for this type of detector. 


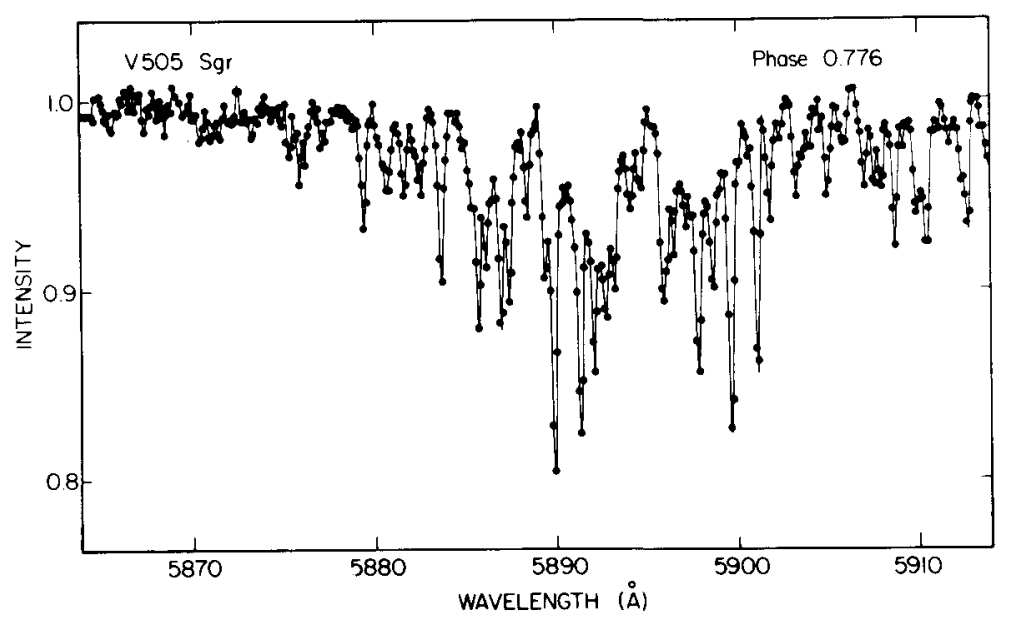

Figure 1. An early September McDonald Reticon observation of the Algol system V505 $\mathrm{Sgr}$ in the neighbourhood of the $\mathrm{NaD}$ lines. The expanded intensity scale shows the top $25 \%$. Numerous telluric water lines obscure the stellar features.

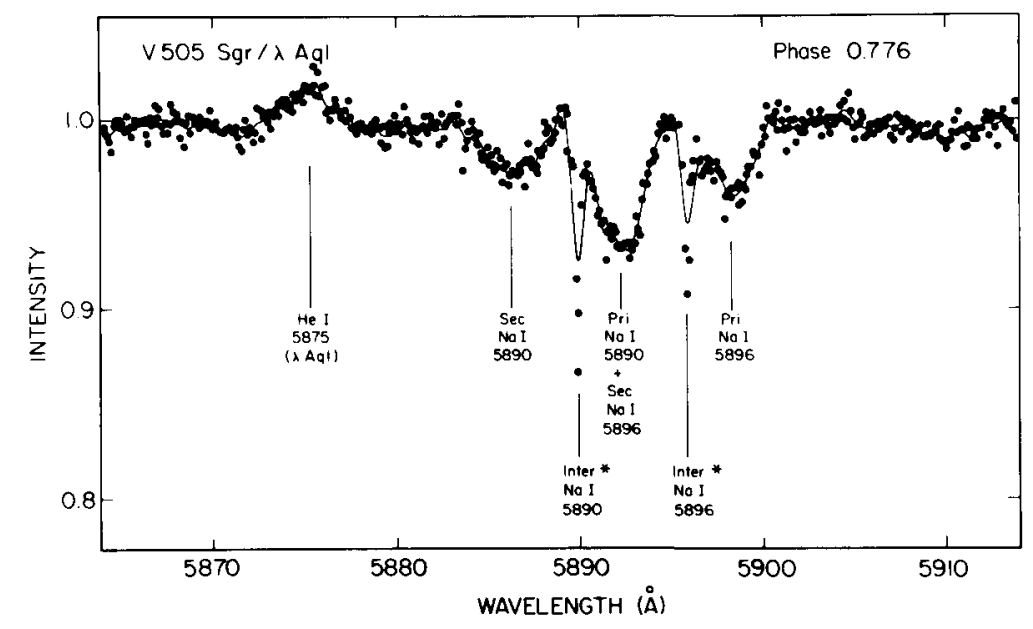

Figure 2. Same observation as Figure 1, except it has been divided by a hot star ( $\lambda$ Aql) observed at a similar airmass instead of a flat-field lamp. The hot star division removes the telluric lines and reveals the presence of the stellar primary and secondary $\mathrm{Na} \mathrm{D}$ lines. The sharp interstellar $\mathrm{NaD}$ lines, which set the wavelength scale, are also identified. The smoothed fit, whose characteristics are determined by the broad stellar features, does not match the much narrower interstellar lines very well. 


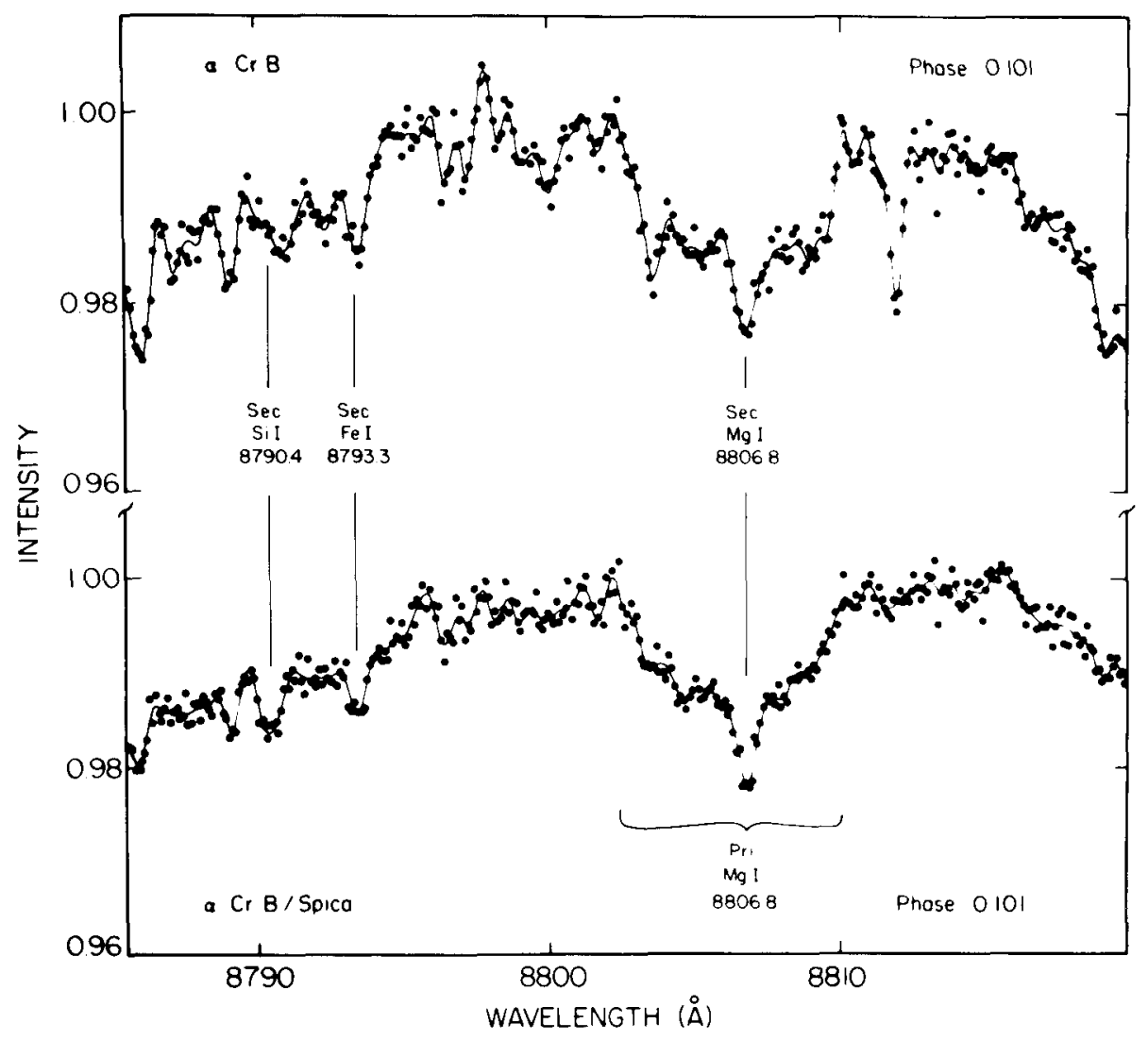

Figure 3. A December McDonald Reticon observation of the detached eclipsing binary $\alpha$ $\mathrm{CrB}$ at $8800 \AA$. The expanded intensity scale shows the top $4 \%$. A very broad primary $\mathrm{MgI}$ line and three secondary lines are identified. In the upper plot telluric water lines, whose widths and strengths are comparable with $\alpha$ CrB's secondary lines, are noticeable. In the lower plot division by a hot star (Spica) instead of a flat-field lamp has removed the telluric lines. This piece of spectrum's location at the high point between the 8750 and $8862 \AA$ Paschen lines gives the "continuum" a curved appearance.

So, to get the most out of photodiode-type detectors in order to track down weak features it is best to make hot-star division a routine, rather than an extraordinary, measure. It may take time and trouble to observe the hot star suited to the particular observational purpose at hand, but it avoids headaches later on.

\section{CNO Abundances In R CMa}

I conclude with the preliminary results of an analysis (Tomkin and Lambert, in preparation) of $\mathrm{CNO}$ abundances in R CMa based on high signal-to-noise-ratio, McDonald 
Reticon observations. Table 1 gives basic information about this Algol system, which is notable for the very low mass $(0.17 \mathrm{mo})$ of its secondary.

\section{TABLE 1}

Basic Data for R CMa

Spectral type (HD)

Spectral type (Sato 1971)

Period (days)

$\mathrm{V}$ (out of eclipse)

$\mathrm{V}$ (primary minimum)

B-V (out of eclipse)
F0

F1V

1.1359

5.74

6.33

0.34

Our investigation concentrates on the primary spectrum and thus is concerned with the $\mathrm{CNO}$ abundances of the primary component. Although the secondary's spectrum has been detected and used to measure its radial-velocity curve (Tomkin 1985), its small contribution to the total light - about $4 \%$ in $\mathrm{V}$ - makes the detection and measurement of secondary atomic or molecular lines suitable for a secondary CNO-abundance analysis impractical.

Selected CI, NI and OI lines between 6,000 and 10,000 $\AA$ were observed. The resolution of the observations was $0.5 \AA$ and each one covered $100 \AA$. Fel and FeII lines included in the observations were used to determine the Fe abundance.

Figure 4 shows three CI lines at $9100 \AA$ A. Residual telluric-line spikes spoil the profiles of two of the lines (9088 and $9094 \AA$ ), but that of the third line (9111 $\AA$ ) is clean and provides a reliable equivalent width. Although these lines are too strong and saturated to provide reliable abundances, nevertheless they demonstrate the presence of primary $\mathrm{CI}$ lines in the spectrum. A much weaker CI line (6587 $\AA$ ) more suitable for abundance analysis is shown in Figure 5.

The measured primary equivalent widths, which are with respect to the continuum of the combined primary and secondary spectra, were then adjusted to be with respect to the continuum of the primary alone. This small, wavelength-dependent correction, which varies between $7 \%$ and $11 \%$, is based on Guinan's (1977) light ratio in V and red and near-infrared colours, representative of R CMa's components, taken from Johnson (1966).

The equivalent widths were analysed by model-atmosphere analysis using Kurucz (1979) flux-constant, main-sequence models. An effective temperature and gravity for the primary of $\mathrm{T}_{\mathrm{e}}=7500 \pm 200 \mathrm{~K}$ and $\log \mathrm{g}=4.1 \pm 0.1$ were adopted. The effective temperature is based on the primary's B-V and $H \beta$ indices (Guinan 1977), which provide temperatures of 7400 and $7500 \mathrm{~K}$, respectively. The gravity is based on a primary mass of 1.1 mo derived from the primary and secondary radial-velocity curves (Tomkin 1985) and a primary radius of $1.57 \mathrm{R}_{\odot}$ derived from Sato's (1971) primary fractional radius of 


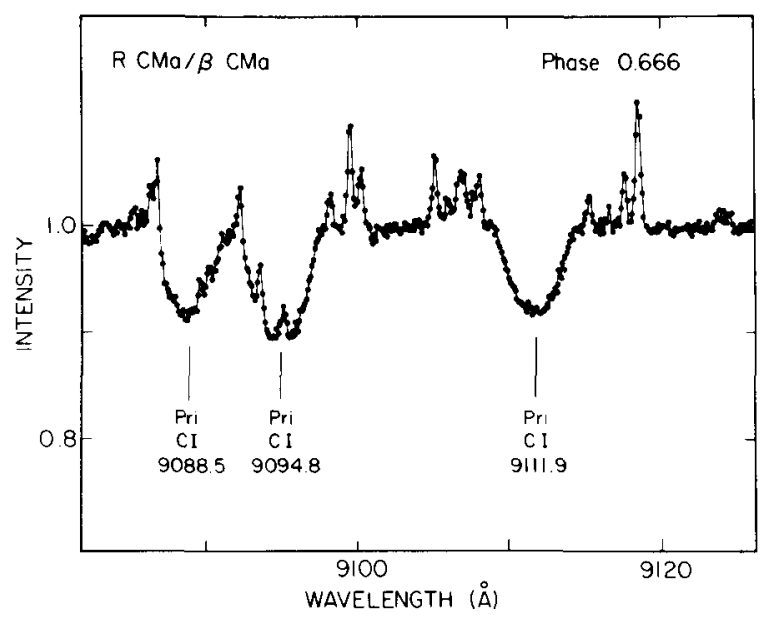

Figure 4. Three CI lines at $9100 \AA$ in the primary of R CMa. Residual telluric lines, which are very strong at this wavelength and which have not been completely removed by the hot-star division, cause the spikes.

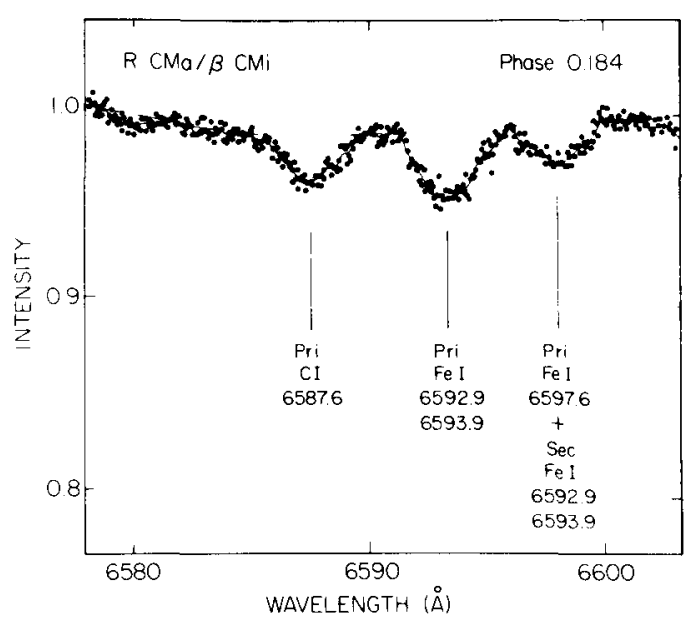

Figure 5: The $6587 \AA \mathrm{C}$ I line in the primary of R CMa. The convolution of the wings of $\mathrm{H} \alpha(6563 \AA)$ in R CMa and the hot star ( $\beta \mathrm{CMi})$ puts a curve in the local continuum, which is marked by the dashed line. 


\section{TABLE 2}

Equivalent Widths and Individual Line Abundances of R CMa's Primary

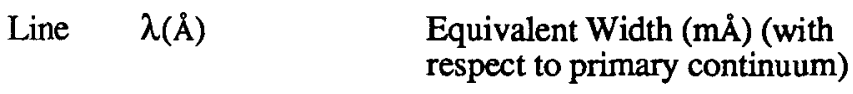

Abundance

$[\mathrm{X} / \mathrm{H}]$

\begin{tabular}{|c|c|c|c|}
\hline $\begin{array}{l}\text { CI } \\
\text { CI }\end{array}$ & $\begin{array}{l}6587.6 \\
9111.9\end{array}$ & $\begin{array}{r}66 \\
316\end{array}$ & $\begin{array}{r}-0.06 \\
---\end{array}$ \\
\hline NI & 7468.3 & 43 & +0.25 \\
\hline $\begin{array}{l}\text { OI } \\
\text { OI } \\
\text { OI }\end{array}$ & $\left.\begin{array}{l}6156.0 \\
6156.8 \\
6158.2\end{array}\right\}$ & 138 & +0.27 \\
\hline $\begin{array}{l}\text { OI } \\
\text { OI } \\
\text { OI }\end{array}$ & $\left.\begin{array}{l}7771.9 \\
7774.2 \\
7775.4\end{array}\right\}$ & 754 & $\cdots$ \\
\hline $\begin{array}{l}\text { SI } \\
\text { SI }\end{array}$ & $\left.\begin{array}{l}8693.9 \\
8694.6\end{array}\right\}$ & 109 & -0.01 \\
\hline $\begin{array}{l}\text { FeI } \\
\text { FeI } \\
\text { FeI } \\
\text { FeI } \\
\text { FeI } \\
\text { FeI } \\
\text { FeI } \\
\text { FeI }\end{array}$ & $\left.\begin{array}{l}6200.3 \\
6546.2 \\
6592.9 \\
6593.9 \\
6633.4 \\
6633.7 \\
6634.1 \\
8327.0\end{array}\right\}$ & $\begin{array}{r}23 \\
67 \\
110\end{array}$ & $\begin{array}{l}-0.07 \\
+0.04 \\
+0.06\end{array}$ \\
\hline $\begin{array}{l}\text { FeII } \\
\text { FeII } \\
\text { FeII }\end{array}$ & $\left.\begin{array}{l}6147.7 \\
6149.2 \\
7449.3\end{array}\right\}$ & 142 & $\begin{array}{l}+0.11 \\
+0.33\end{array}$ \\
\hline
\end{tabular}

0.325. Guinan's (1977) almost identical primary fractional radius of 0.327 leads to the same gravity.

A microturbulence of $2 \mathrm{~km} \mathrm{~s}^{-1}$ is obtained from the requirement that the FeI lines yield $\mathrm{Fe}$ abundances that are not equivalent-width dependent. The same requirement on the CI line-based $C$ abundances yields a $6 \mathrm{~km} \mathrm{~s}^{-1}$ microturbulence. We adopted the lower FeI line-based microturbulence. The 6587 and $9111 \AA$ lines are the only two CI lines for which we have reliable equivalent widths and the very large $316 \mathrm{~m} \AA$ equivalent width of the $9111 \AA$ line, which is the stronger line of the two, makes it prone to significant nonLTE effects. 
Table 2 gives the equivalent widths and individual line abundances for the CI, NI, OI, SI, FeI and FeII lines. Table 3 summarises the abundance results. The parenthesised numbers, which give the results for $4 \mathrm{~km} \mathrm{~s}^{-1}$ microturbulence instead of the $2 \mathrm{~km} \mathrm{~s}^{-1}$ adopted, indicate the microtubulence dependence.

\section{TABLE 3}

Abundances of R CMa's Primary

$\frac{\text { Abundance }}{[\mathrm{X} / \mathrm{Fe}]}$

\begin{tabular}{lccc}
\hline $\mathrm{C}$ & $-0.06(-0.18)$ & -0.20 & $(-0.11)$ \\
$\mathrm{N}$ & $+0.25(+0.13)$ & $+0.11(+0.20)$ \\
$\mathrm{O}$ & $+0.27(+0.16)$ & +0.13 & $(+0.23)$ \\
$\mathrm{S}$ & $-0.01(-0.17)$ & -0.15 & $(-0.10)$ \\
$\mathrm{Fe}\{\mathrm{FeI}$ & $+0.05(-0.16)$ & -- & --- \\
& $+0.22(+0.01)$ & -- & -- \\
\hline
\end{tabular}

Results are for the adopted $2 \mathrm{~km} \mathrm{~s}^{-1}$ microturbulence; results in parentheses are for a $4 \mathrm{~km}$ $\mathrm{s}^{-1}$ microturbulence.

The FeI and FeII line-based Fe abundances differ by 0.17 dex, a difference which probably is not significant in view of the scatter of the individual abundances (see Table 2). The Fe abundance suggests that R CMa is marginally metal rich, as does the $\Sigma(\mathrm{C}+\mathrm{N}+$ O) abundance. Except for a possible deficiency of C, the CNO distribution is essentially solar. The $\mathrm{C}$ deficiency $([\mathrm{C} / \mathrm{Fe}]=-0.2 ;[\mathrm{C} / \mathrm{N}]=-0.3)$ is of marginal significance because the CNO results are based on only one line of each element. How does this CNOabundance distribution, which may be moderately, but is not greatly, different from solar, compare with theoretical expectations?

R CMa's primary and secondary have masses of 1.1 and $0.17 \mathrm{~m}_{\odot}$ (Tomkin 1985). The secondary's low mass and the assumption that originally it must have been the more massive component argue that it has suffered extensive Roche-lobe-overflow-related mass loss and that a major part of the mass lost may have ended up on the present primary. At the time of Roche-lobe overflow the secondary would most likely have been a 1 to $2 \mathrm{~m}_{\mathcal{O}}$ star in a late main-sequence or early red-giant stage of evolution. Prior main-sequence CNO cycling would already have altered the star's composition so that over the central one-third, by mass, most $\mathrm{C}$ would have become $\mathrm{N}$ and over the central one-tenth, by mass, half of the $\mathrm{O}$ would also have become $\mathrm{N}$. Thus some of the material transferred to the present primary may have had the $\mathrm{C}$ deficiency, $\mathrm{N}$ richness and moderate $\mathrm{O}$ deficiency characteristic of partial CNO cycling. Do the observed primary CNO abundances exclude this possible contamination?

We consider two extreme scenarios: completely conservative, and completely nonconservative, mass transfer. For the case of fully conservative mass transfer we use the requirement that the secondary (i.e., the original primary) must have been massive enough 
to complete its main-sequence evolution during the lifetime of the disk and VandenBerg's (1985) models to set a lower limit of $1.1 \mathrm{~m} \odot$ on its initial mass. This leaves room for a companion (now the primary) with an initial mass of $0.2 \mathrm{mo}$. The low initial mass of the companion means that these limits on the components' initial masses cannot be very different from the actual initial masses in the fully conservative case. The observed 1.1 and 0.17 mo primary and secondary masses then lead to the conclusion that in the primary we see a star that was $0.2 \mathrm{~m}_{\odot}$ and has gained $0.9 \mathrm{~m}_{\odot}$ and in the secondary we see a star that was $1.1 \mathrm{~m} \odot$ and has lost $0.9 \mathrm{~m} \odot$. Thus in this fully conservative scenario the secondary has indeed suffered extensive mass loss - the outer $82 \%$ of its mass has been transferred to the primary.

The part of this transferred material that was exposed to CNO cycling before the onset of mass transfer is, however, surprisingly small. Dearborn, Eggleton and Schramm's (1976) composition profiles for a $1 \mathrm{m \odot}$, solar-abundance model near the end of its main-sequence evolution show that $\mathrm{C}$ is $\mathrm{CNO}$-cycle depleted in only $20 \%$ of the transferred material and $\mathrm{O}$ is completely unaffected. If the mass transfer did not cause large-scale mixing, i.e. it turned the composition gradient of the transferred material inside-out, then the material presently on the surface of the primary would be extremely non-solar $([\mathrm{C} / \mathrm{Fe}]=-2.4$, $[\mathrm{N} / \mathrm{Fe}]=+0.8$ and $[\mathrm{O} / \mathrm{Fe}]=0.0$ for an assumed initial solar-CNOFe distribution).

This very $\mathrm{C}$-deficient composition is not confirmed by the observed abundances $([\mathrm{C} / \mathrm{Fe}]$ $=-0.2,[\mathrm{~N} / \mathrm{Fe}]=+0.1$ and $[\mathrm{O} / \mathrm{Fe}]=+0.1)$. The $\mathrm{CNO}$ abundances of $\mathrm{R} \mathrm{CMa}$ 's primary thus appear to exclude the conservative-mass-transfer, no-mixing scenario.

On the other hand if the transferred material is fully mixed, then the primary's predicted surface abundances show only modest departures from the solar mixture: $[\mathrm{C} / \mathrm{Fe}]=-0.1$, $[\mathrm{N} / \mathrm{Fe}]=+0.3$ and $[\mathrm{O} / \mathrm{Fe}]=0.0$. These changes are at the same level as the uncertainty $(\sim 0.3$ dex $)$ in the observed $\mathrm{CNO}$ abundances. Thus although the similarity between the -0.4 dex predicted $[\mathrm{C} / \mathrm{N}]$ and -0.3 dex observed $[\mathrm{C} / \mathrm{N}]$ is suggestive, the predicted changes for the conservative-mass-transfer, with-mixing case are not large enough to make it observationally distinctive.

In the alternative extreme scenario of completely non-conservative mass transfer all the mass lost by the present-day secondary is lost from the system; none of it is transferred to the primary. Thus the primary's surface composition would retain its assumed initial solar-CNO Fe distribution. This is quite consistent with the observed abundances, which are solar to within their $\sim 0.3$ dex observational error.

We conclude that $\mathrm{R}$ CMa's primary CNO abundances exclude the possibility of conservative mass transfer without mixing, but do not allow us to decide between conservative mass transfer with mixing and completely non-conservative mass transfer.

\section{Conclusions}

High signal-to-noise ratio spectra have opened two new avenues for exploration of Algol systems: detection of faint secondaries and measurement of the weak lines most suitable for abundance determinations. Detection of the secondary spectrum and measurement of its radial-velocity curve is often the last step in the determination of the system's dimensions and masses of its components. Abundances, particularly the CNO abundances, can be a useful check on evolutionary status.

Algol is a good example of weak secondary spectrum detection. Before the advent of modern detectors only the B8 primary spectrum was visible, along with the third component's spectrum during primary eclipse. Reticon observations (Tomkin and Lambert 1978) revealed not only the secondary spectrum, but also that of the third component outside of primary eclipse; in effect Algol changed from a single-lined spectroscopic binary into a spectroscopic triple. 
$\beta$ Lyrae is an example of instructive CNO abundances. A Reticon-based abundance analysis of the primary (Balachandran et al. 1986) shows a distribution characteristic of extreme CNO cycling, which confirms the modern picture of $\beta$ Lyrae's primary as the exposed core of an originally much more massive star. We note that, although this study put a low limit on $\beta$ Lyrae's $C$ abundance, the actual $C$ abundance is still unknown because there are no detectable C lines in the visible or near-infrared. C lines (C I, C II, C III and C IV) do inhabit the extreme ultraviolet (Plavec 1985; Mazzali 1987), however, and will eventually provide the $\mathrm{C}$ abundance.

I am much less naive than I used to be, but I am still naive enough to think that we have only scratched the surface of what can be done with high signal-to-noise ratio observations.

This research has been supported, in part, by the National Science Foundation of the United States of America through grant AST 86-14423.

\section{References}

Balachandran, S., Lambert, D. L., Tomkin, J., and Parthasarathy, M. 1986, M.N.R.A.S., 219, 479.

Dearborn, D. S. P., Eggleton, P. P., and Schramm, D. N. 1976, Ap. J., 203, 455.

Guinan, E. F. 1977, A. J., 82, 51.

Johnson, H. L. 1966, Ann. Rev. Astr. Ap. , 4, 196.

Kurucz, R. L. 1979, Ap. J. Suppl., 40, 1.

Mazzali, P. A. 1987, Ap. J. Suppl., 65, 695.

Plavec, M. J. 1985, in "Interacting Binaries", ed. P. P. Eggleton and J. E. Pringle (Dordrecht; Reidel), p. 155.

Sato, K. 1971, Pub. Astr. Soc. Japan, 23, 335.

Tomkin, J. 1985, Ap. J., 297, 250.

Tomkin, J. and Lambert, D. L. 1978, Ap. J. Letters, 222, L119.

Tomkin, J. and Popper, D. M. 1986, A. J., 91, 1428.

VandenBerg, D. A. 1985, Ap. J. Suppl., 58, 711.

Vogt, S. S., Tull, R. G. and Kelton, P. 1978, Appl. Opt., 17, 574. 


\section{DISCUSSION}

Hilditch suggested a fourth possibility for the evolution of $R$ CMa. If the original primary, of about $1 \mathrm{~m}_{\Theta}$, lost angular momentum by magnetic braking, the orbital period would be reduced and its Roche lobe would become as small as the star itself. Mass would then be transferred before any significant changes to CNO abundances had been made. Budding felt that this was an ad hoc hypothesis, but that $R$ CMa was sufficiently unusual that perhaps any explanation of it would appear to be such an hypothesis.

Bolton agreed that the use of hot stars as standards to remove telluric lines and to "flat-field" Reticon spectra was of ten desirable, but emphasized the importance of observing the standard stars at the same air-mass as the programme stars and of taking care that both stars illuminated the collimator in the same way. Tomkin replied that careful choice of standards guarantees the first condition and observation of the standards during the same observing run as, and with the same spectrograph settings used for, the programme stars guarantees the second. Bolton also noted that sometimes we need simultaneous observations of widely spaced lines (e.g. the lower Balmer lines) and photographic plates still have advantages over electronic detectors for this purpose. A signal-to-nolse ratio of up to about 150 can be achieved when finegrain emulsions are exposed to densities greater than 1.0 or 1.2 . Later in the discussion, Hill mentioned that Reticon spectra of Vega had recently been multiplexed by Cowley and Gulliver, to cover the spectral region from $3000 \AA$ to $8000 \AA$. 